\title{
Calculation and Simulation Study on Transient Stability of Power System Based on Matlab/Simulink
}

\author{
Xiu Feng $\mathrm{SHI}^{1}$, Yan Rong YANG ${ }^{1}$ and Jun Feng WANG ${ }^{1}$
}

${ }^{1}$ School of Information Technology and Engineering Yuxi Normal University, Yuxi , Yunnan , 653100

\begin{abstract}
The stability of the power system is destroyed, will cause a large number of users power outage, even cause the collapse of the whole system, extremely serious consequences. Based on the analysis in single machine infinite system as an example, when at the f point two phase ground fault occurs, the fault lines on either side of the circuit breaker tripping resection at the same time,respectively by two kinds of calculation and simulation methods of system transient stability analysis, the conclusion are consistent. and the simulation analysis is superior to calculation analysis
\end{abstract}

Keywords. Matlab, power system, transient; stability, simulation

\section{Introduction}

The failure in stability of the power system would cause power outage to many users or even collapse of the whole system, thus bringing very serious consequences. Therefore, the study on the stability of the power system is of great significance in ensuring safe and reliable operation of the power system.

After the power system is subject to a large disturbance, the imbalance between mechanical torque and electromagnetic torque on the generator rotor leads to a change in the relative position between synchronous generator rotors, i.e. the current, voltage and electromagnetic power of the system are changed as the relative angle between generator potentials is changed. The study on the transient stability of the power system refers to researching whether parallel operated synchronous generators can still operate synchronously and the load is normal after the power system is subject to a large disturbance in an operation mode. The reasons for the large disturbance mainly include: short circuit fault; turning on or off main components of the system, such as generator, transformer and line; sudden change in load, such as addition of large users. The short-circuit fault is usually used to check the transient stability of the system as it is the most severe among them.

\section{Calculation of transient stability of power system}

Taking the diagram of single-machine infinite bus system shown in Fig. 1 as an example, the transient stability of the power system is calculated after the faulty line is cut off through simultaneous tripping of circuit breakers on both sides of the line in the case of two-phase ground fault at the point $\mathrm{f}$. 


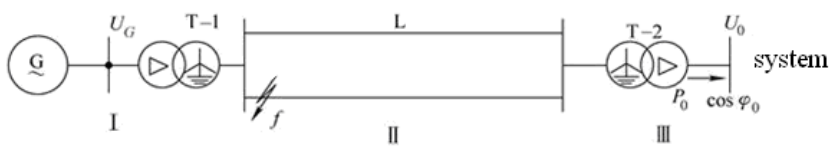

Fig. 1 Diagram of single-machine infinite bus system

The parameters of components in the single-machine infinite bus system shown in Fig. 1 are as follows $^{[1]}$ :

Parameters of the generator $\mathrm{G}$ :

$P_{G N}=300 \mathrm{MW}, \quad U_{G N}=10.5 \mathrm{KV}, \quad \cos =0.85, \quad X_{d}=X_{q}=2.36$

$X_{d}^{\prime}=0.32, \quad X_{d}^{\prime \prime}=0.252, \quad X_{q}^{\prime \prime}=0.243, \quad X_{2}=0.243, \quad T_{j}=6 s$

Parameters of the transformer T-1: $\mathrm{S}_{\mathrm{TN} 1}=360 \mathrm{MVA}$, $\mathrm{U}_{\mathrm{ST} 1} \%=14 \%, \mathrm{~K}_{\mathrm{T} 1}=10.5 / 242$;

Parameters of the transformer T-2: $\mathrm{S}_{\mathrm{TN} 2}=360 \mathrm{MVA}$, $\mathrm{U}_{\mathrm{ST} 2} \%=14 \%, \mathrm{~K}_{\mathrm{T} 2}=220 / 121$;

Parameters of the transmission line $\mathrm{L}: \mathrm{U}_{\mathrm{N}}=220 \mathrm{KV}$, $1=200 \mathrm{~km}, \mathrm{X}_{\mathrm{L}}=0.41 \Omega / \mathrm{km}, \mathrm{X}_{\mathrm{L} 0}=4 \mathrm{X}_{\mathrm{L}}$;

Operation: $\mathrm{U}_{0}=115 \mathrm{KV}, \mathrm{P}_{0}=250 \mathrm{MW}, \cos \varphi_{0}=0.98$.

\subsection{Calculation of network parameters and operating parameters}

$\mathrm{S}_{\mathrm{B}}=250 \mathrm{MVA}, \mathrm{U}_{\mathrm{B} I I I}=115 \mathrm{KV}, U_{B \mathrm{X}}=U_{\mathrm{X}} \quad \mathrm{U}_{\mathrm{B} \text { II }}=\mathrm{U}_{\mathrm{B} I I I}$

$\times \mathrm{K}_{\mathrm{T} 2}=115 \times 220 / 121=209 \mathrm{KV}$,

$\mathrm{U}_{\mathrm{BI}}=\mathrm{U}_{\mathrm{B} \text { II }} \times \mathrm{K}_{\mathrm{T} 1}=209 \times 10.5 / 242=9.07 \mathrm{KV}$.

1.1.1 Per unit values are as follows after reduction of component parameters:

$$
\begin{aligned}
& X_{d}=X_{q}=2.36 \times \frac{250 \times 0.85}{300} \times\left(\frac{10.5}{9.07}\right)^{2}=2.241 \\
& X_{d}^{\prime}=0.32 \times \frac{250 \times 0.85}{300} \times\left(\frac{10.5}{9.07}\right)^{2}=0.304 \\
& X_{T 1}=0.14 \times \frac{250}{360} \times\left(\frac{242}{209}\right)^{2}=0.130 \\
& X_{L}=0.41 \times 200 \times \frac{250}{209^{2}}=0.47 \\
& X_{L 0}=4 \times X_{L}=0.47 \times 4=1.88 \\
& X_{T 2}=0.14 \times \frac{250}{360} \times\left(\frac{220}{209}\right)^{2}=0.108 \\
& X_{2}=0.23 \times \frac{250 \times 0.85}{300}\left(\frac{242}{209}\right)^{2}=0.218 \\
& T_{J}=6 \times \frac{300}{0.85 \times 250}=8.46 s
\end{aligned}
$$

The equivalent circuit during the normal operation of the system is shown in Fig. 2a), and integrated impedances of the system are as follows:

$$
\begin{aligned}
& X_{T L}=X_{T 1}+\frac{1}{2} X_{L}+X_{T 2}=0.130+\frac{1}{2} \times 0.47+0.108=0.473 \\
& X_{d \Sigma}=X_{q \Sigma}=X_{d}+X_{T L}=2.241+0.473=2.714 \\
& X_{d \Sigma}^{\prime}=X_{d}^{\prime}+X_{T L}=0.304+0.473=0.777
\end{aligned}
$$

1.1.2 Calculation results of per unit values of operating parameters are as follows:

$P_{0}=\frac{P_{0}}{S_{B}}=\frac{250}{250}=1, \quad U_{0}=\frac{U_{0}}{U_{B X I}}=\frac{115}{115}=1$,

$Q_{0}=P_{0} \tan { }_{0}=P_{0} \tan \left(\cos ^{1} 0.98\right)=0.2$

$E_{0}=\sqrt{\left(U_{0}+\frac{Q_{0} X_{d \Sigma}^{\prime}}{U_{0}}\right)^{2}+\left(\frac{P_{0} X_{d \Sigma}^{\prime}}{U_{0}}\right)^{2}}=\sqrt{(1+0.2 \times 0.777)^{2}+(1 \times 0.777)^{2}}=1.392$

$\delta_{0}=\tan ^{-1} \frac{\frac{P_{0} X_{d \Sigma}^{\prime}}{U_{0}}}{U_{0}+\frac{Q_{0} X_{d \Sigma}^{\prime}}{U_{0}}}=\tan ^{-1} \frac{0.777}{1+0.2 \times 0.777}=33.92^{\circ}$

\subsection{Calculation of transferring impedance and power characteristic of the system}

The equivalent circuit during the normal operation of the system, negative sequence and zero sequence equivalent networks in the case of two-phase short circuit at the point $\mathrm{f}$, equivalent network in the case of short circuit and equivalent network after clearing of short circuit are shown in a), b), c), d) and e) in Fig. 2 respectively.

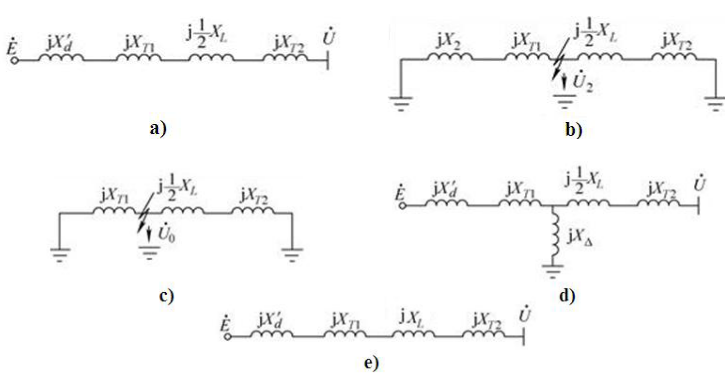

Fig. 2

a) Equivalent circuit during the normal operation b) Negative sequence network c) Zero sequence network

d) Equivalent network in the case of short circuit e) Equivalent network after clearing of short circuit

As seen in b) negative sequence network and c) zero sequence network in Fig. 2, the negative sequence and zero sequence equivalent reactance at the short circuit 
point are:

$$
\begin{aligned}
X_{2 \Sigma}= & \frac{\left(X_{2}+X_{T 1}\right)\left(\frac{1}{2} X_{L}+X_{T 2}\right)}{X_{2}+X_{T 1}+\frac{1}{2} X_{L}+X_{T 2}} \\
= & \frac{(0.218+0.130)\left(\frac{1}{2} \times 0.47+0.108\right)}{(0.218+0.130)+\left(\frac{1}{2} \times 0.47+0.108\right)} \\
= & 0.173
\end{aligned}
$$

$$
X_{0 \Sigma}=\frac{X_{T 1}\left(\frac{1}{2} X_{L 0}+X_{T 2}\right)}{X_{T 1}+\frac{1}{2} X_{L 0}+X_{T 2}}=\frac{0.130 \times\left(\frac{1}{2} \times 1.88+0.108\right)}{0.130+\frac{1}{2} \times 1.88+0.108}=0.116
$$

The additional reactance at the short circuit point in the case of two-phase grounding short circuit is:

$X_{\Delta}=\frac{X_{0 \Sigma} X_{2 \Sigma}}{X_{0 \Sigma}+X_{2 \Sigma}}=\frac{0.173 \times 0.116}{0.173+0.116}=0.069$

From d) equivalent network in the case of short circuit in Fig. 2, the transferring impedance and power characteristic of the system are:

$$
\begin{aligned}
& X_{I I}=X_{d}^{\prime}+X_{T 1}+\frac{1}{2} X_{L}+X_{T 2}+\frac{\left(X_{d}^{\prime}+X_{T 1}\right)\left(\frac{1}{2} X_{L}+X_{T 2}\right)}{X_{\Delta}} \\
& =0.434+0.343+\frac{0.434 \times 0.343}{0.069} \\
& =2.93
\end{aligned}
$$$$
P_{I I}=\frac{E_{0} U_{0}}{X_{I I}} \sin \delta=\frac{1.392 \times 1}{2.93} \sin \delta=0.48 \sin \delta
$$

From e) equivalent network after clearing of short circuit in Fig. 2, the transferring impedance and power characteristic of the system are:

$X_{I I I}=X_{d}^{\prime}+X_{T 1}+X_{L}+X_{T 2}=0.304+0.130+0.47+0.108=1.102$

$P_{I I I}=\frac{E_{0} U_{0}}{X_{I I I}} \sin \delta=\frac{1.392 \times 1}{1.102} \sin \delta=1.38$

\subsection{Calculation of limit clearing angle}

The limit clearing angle $\delta_{c m}$ is calculated as follows through the equal area criterion [1]:

$$
\begin{aligned}
\delta_{c m} & =\cos ^{-1} \frac{P_{0}\left(\delta_{h}-\delta_{0}\right)+P_{I I I M} \cos \delta_{h}-P_{I I M} \cos \delta_{0}}{P_{I I I M}-P_{I I M}} \\
& =\cos ^{-1} \frac{1 \times \frac{\pi}{180^{0}}\left(133.6^{0}-33.92^{0}\right)+1.38 \cos 133.6^{0}-0.48 \cos 33.92^{0}}{1.38-0.48} \\
& =1.122
\end{aligned}
$$

Where, the critical angle $\delta_{h}$ is

$\delta_{h}=180^{\circ} \quad \sin ^{1} \frac{P_{0}}{P_{\text {IIIM }}}=180^{\circ} \quad \sin ^{1} \frac{1}{1.38}=133.6^{\circ}$

$\delta_{c m}$ is converted to degrees: $\delta_{c m}=1.122 \times \frac{180^{\circ}}{\pi}=64.3^{\circ}$

\subsection{Calculation of generator rocking curve $\delta-t$}

In the simple system above, the generator rocking curve $\boldsymbol{\delta}-\boldsymbol{t}$ (i.e. motion equation of rotor) during the fault is

$$
\left\{\begin{array}{l}
\frac{d \delta}{d t}=(\omega-1) \omega_{0} \\
\frac{d \omega}{d t}=\frac{1}{T_{j}}\left(P_{T}-\frac{E U}{X_{I I}} \sin \delta\right)
\end{array}\right.
$$

These are two first-order non-linear ordinary differential equations with known initial conditions, that is

$t=0 ; \omega=1 ; \delta=\delta_{0}=\sin ^{1} \frac{P_{T}}{P_{I M}}$.

After calculating the curve $\delta-t$ during the fault, the limit clearing time corresponding to the limit clearing angle can be found from the curve.

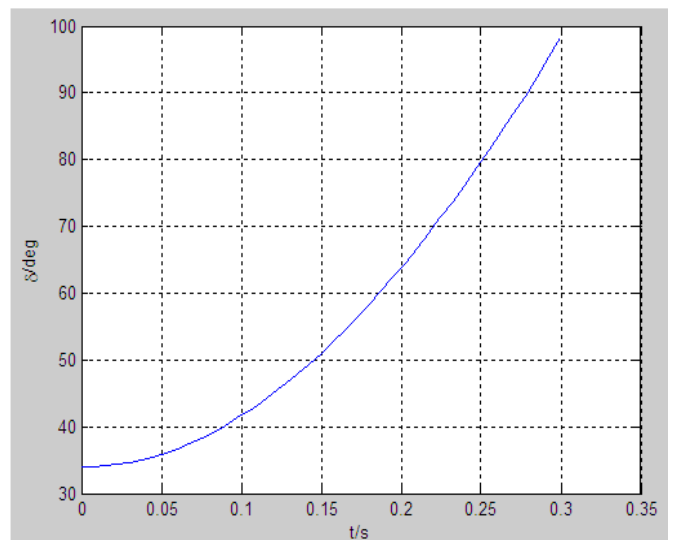

Fig. 3 Curve $\boldsymbol{\delta}-\boldsymbol{t}$ during system fault

It is very difficult to conduct the analytical solution to two simple first-order non-linear ordinary differential equations (1-1). In this paper, the generator rocking curve $\delta-t$ during the fault is solved with Ronge-Kuta method in Matlab ${ }^{[2}$.] After running the program, the curve $\delta-t$ during the system fault is obtained, as shown in Fig. 3. The limit clearing time corresponding to the limit clearing angle $\delta_{c m}=64.3^{0}$ obtained from Fig. 3 (or output result) is $0.2 \mathrm{~s}$. 
If the clearing time is known, the curve $\boldsymbol{\delta}-\boldsymbol{t}$ needs to be calculated to determine the stability of the system. When the fault clearing is calculated through the curve, another differential equation as shown below needs to be solved as changes in system parameters can lead to a change in the power characteristic of the generator

$$
\left\{\begin{array}{l}
\frac{d \delta}{d t}=(\omega-1) \omega_{0} \\
\frac{d \omega}{d t}=\frac{1}{T_{J}}\left(P_{T}-\frac{E^{\prime} U}{X_{I}} \sin \delta\right)
\end{array}\right.
$$

Initial conditions for the equation 1-2 are $t_{c}$, where $\boldsymbol{t}_{c}$ is the given clearing time, $\delta_{c}$ and $\omega_{c}$ are $\delta$ and $\boldsymbol{\omega}$ corresponding to $\boldsymbol{t}_{\boldsymbol{c}}$. $\boldsymbol{\delta}_{c}$ and $\boldsymbol{\omega}_{c}$ can be obtained from the curve $\delta-t$ and $\boldsymbol{\omega}-\boldsymbol{t}$ during the fault. The curve of $\delta$ and $\omega$ as a function of time can be obtained from the equation 1-2. Generally, the system is stable transiently if $\boldsymbol{\delta}$ is always no more than $180^{\circ}$ and the vibration amplitude value is becoming smaller and smaller in a few seconds of calculation.

It is very difficult to conduct the analytical solution to two simple first-order non-linear ordinary differential equations (1-2). The generator rocking curve $\delta-t$ after clearing of the system fault is solved with Ronge-Kuta method in Matlab. If the clearing time is $0.1 \mathrm{~s}$, the curve $\delta-t$ during the system fault obtained is shown in Fig. 4 after modifying system parameters and running the program. Fig. 4 (or output result) shows that reduction begins at $0.5455 \mathrm{~s}$ and the

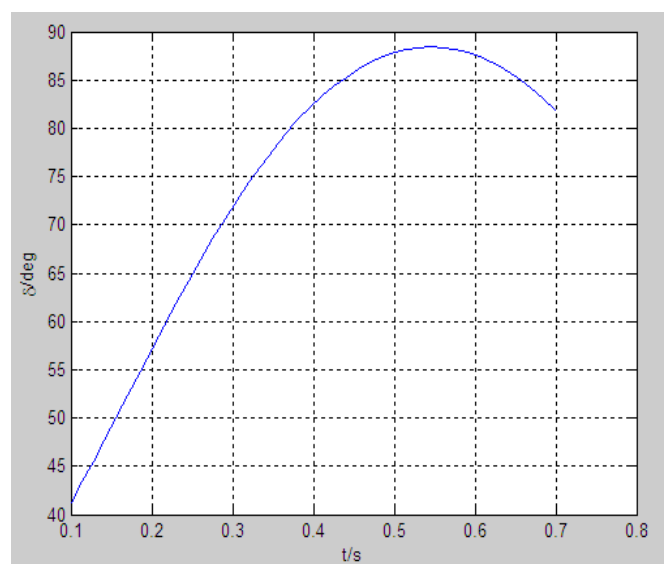

Fig. 4 Curve $\delta-t$ after clearing of system fault maximum angle is $\delta=88.44^{\circ}$. Therefore, the system is stable transiently.

\section{Establishment of Simulation Model of Transient Stability}

The simulation model ${ }^{[2]}$, as shown in Fig. 5, is established to study the transient stability according to the single-machine infinite bus system in Fig. 1.

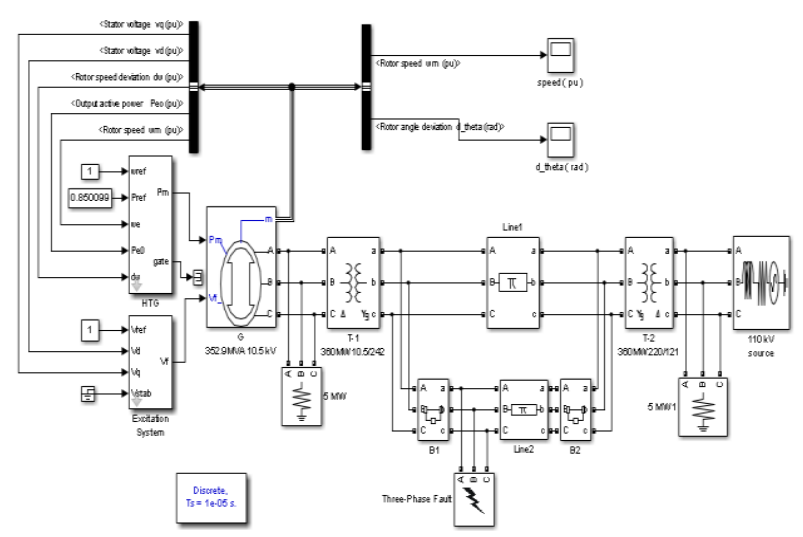

Fig. 5 simulation model of transient stability of power system

On the simulation diagram, parameters of the generator, transformer, transmission line, infinite bus system, circuit breaker B1 and B2 are set according to the given conditions, and then the generator is initialize $d$ with Powergui module ${ }^{[3]}$. The generator bus is set to $\mathrm{PV}$ bus, the terminal voltage is set to $18 \mathrm{KV}$, the output power is set to 300MV.A and then

the system load flow is updated; the sampling time is set to $1 \times 10^{-5} \mathrm{~s}$. Finally, simulation parameters are set, the discrete algorithm is chosen, the start time for simulation is set to 0 , and the end time is set to $5 \mathrm{~s}$. In the fault point module, the system is set to have $\mathrm{BC}$ two-phase grounding short circuit at $0.05 \mathrm{~s}$ and cut off the short circuited line at $0.1 \mathrm{~s}$ after the fault ${ }^{[4]}$.

\section{Analysis on simulation result}

The speed change curve, as shown in Fig. 6, of the generator is obtained through simulation according to the simulation model Fig. 5. The parameter settings of the circuit breaker module B1 and B2 are changed to cut off the line at $0.5 \mathrm{~s}$ after the fault. The speed change curve, as shown in Fig. 7, of the generator is obtained through the second simulation. 


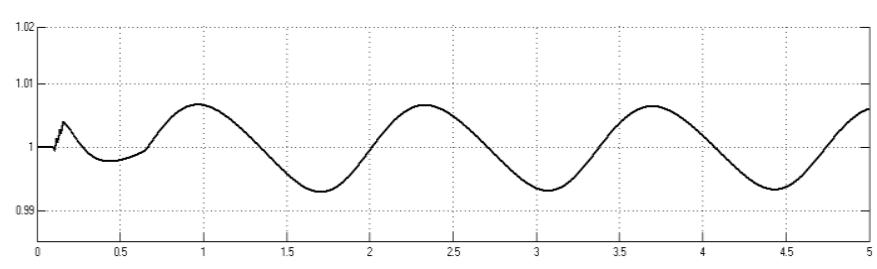

Fig. 6 Speed change curve of generator when cutting off the line at $0.1 \mathrm{~s}$ after the fault

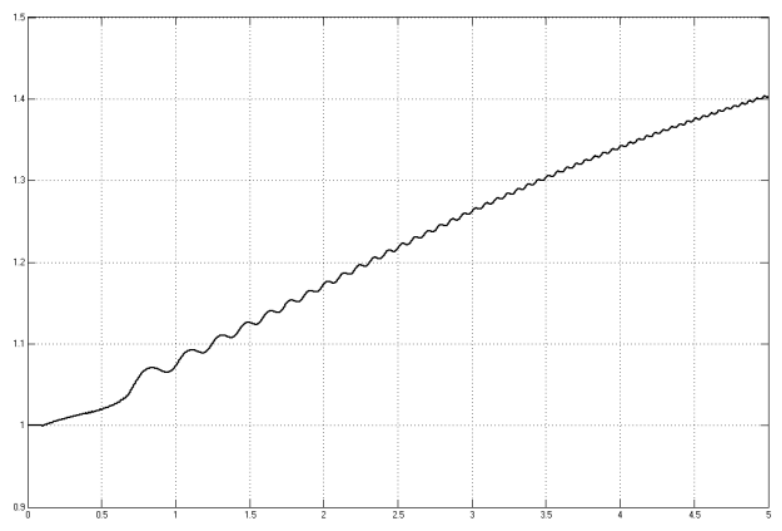

Fig. 7 Speed change curve of generator when cutting off the line at $0.5 \mathrm{~s}$ after the fault

The simulation curve in Fig. 6 shows that the speed of the generator gradually decreases with the increase of the time (variation range: $0.99 \sim 1.01$ ) and tends to become stable constants in the case of two-phase grounding short circuit fault at the point $f$ after cutting off the faulty line after $0.1 \mathrm{~s}$ (the clearing time is less than the limit clearing time), therefore the system is stable; the simulation curve in Fig. 7 shows that the speed of the generator gradually increases with the increase of the time in the case of two-phase grounding short circuit fault at the point $\mathrm{f}$ after cutting off the faulty line after $0.5 \mathrm{~s}$ (the clearing time is greater than the limit clearing time), therefore the system is unstable

\section{Conclusion}

To sum up, the limit clearing angle obtained by calculating the transient stability of the power system is $64.3^{\circ}$, and the limit clearing time obtained with Ronge-Kuta method in Matlab is $0.2 \mathrm{~s}$ based on the limit clearing angle. If the clearing time is $0.1 \mathrm{~s}$ (less than the limit clearing time), the maximum angle $\delta$ obtained with Ronge-Kuta method in Matlab is $88.44^{\circ}$, which is less than $180^{\circ}$, and the vibration amplitude value is becoming smaller and smaller, therefore the system is stable transiently.and for power system transient stability simulation analysis obtained the system is also stable transiently as the speed of the generator gradually decreases with the increase of the time after cutting off the faulty line at $0.1 \mathrm{~s}$ after the fault. Therefore, the conclusions are consistent In comparison, the simulation analysis is superior to calculation, the reason is the complicated calculation can be avoided.

\section{References}

[1] Li Guangqi, Transient Analysis of Power System [M], Beijing: China Electric Power Press, 2007

[2] Yu Qun, MATLAB/Simulink Power System Modeling and Simulation [M], Beijing: China Machine Press. 05 (2011)

[3] Zhao Haibin, MATLAB Applications [M], Beijing: Tsinghua University Press, 05 (2012)

[4] Zhou Junjie, Matlab/Smiulink Example Explanation [M], Beijing: China WaterPower Press, 05 (2014) 